\title{
CROSSING ALBERTA
}

Under so much sky every object is a portent;

the farmhouse standing by itself, the shacks and barns clustering,

slanted by the wind, the one tree leaning

across the prairie.

The yellow ground rises

slightly, and slopes

away, out of sight or reach.

I am surrounded by my own track, immersed in my own journey.

The prairie pulls all its outgrowths toward itself, it is one color. The traveller at the window

of a speeding vehicle looks away, inside, fearing the heat, fearing the dazzled mind's

astigmatism, the light on these yellow fields, the horizon, like the edge of his memory. 